\title{
Synaptic Plasticity in the Molluscan Peripheral Nervous System: Physiology and Role for Peptides ${ }^{1}$
}

\author{
C. J. COATES AND A. G. M. BULLOCH${ }^{2}$ \\ Department of Medical Physiology, Health Sciences Center, University of Calgary, Calgary, Alberta, Canada T2N 4N1
}

\begin{abstract}
The plasticity of a synapse in the molluscan peripheral nervous system was examined under a variety of experimental, physiological, and pharmacological conditions. These studies employed the isolated salivary glands and attached buccal ganglia of the freshwater snail Helisoma. Action potentials evoked in buccal neuron 4 normally evoke a large excitatory postsynaptic potential (EPSP) which drives an action potential in gland secretory cells. In order to measure modulation of the EPSP, action potential generation in gland cells was prevented by bathing the preparation in low calcium, high magnesium salines. The relationship between the gland EPSP amplitude and specific physiological properties of neuron 4 was analyzed. In common with some central molluscan synapses, the EPSP was found to be strongly influenced by the membrane potential of neuron 4 . Specifically, its amplitude was reduced by hyperpolarization of the neuron 4 soma. The relationship between EPSP amplitude and somatic potential of neuron 4 was linear in the range from resting potential $(-47 \pm 6 \mathrm{mV})$ to $-100 \mathrm{mV}$. Furthermore, the EPSP amplitude was directly proportional to the action potential half-width of neuron 4 . In order to evaluate the possible physiological role of this action potential/EPSP relationship, we examined whether gland EPSPs are modulated during the spike broadening that occurs in both spontaneous burst activity and imposed impulse trains. The preceding action potential/EPSP relationship was maintained under both of these conditions, i.e., EPSP magnitude increased as spikes broadened during bursts or trains. The peptidergic modulation of neuroglandular transmission was also examined. The molluscan peptide $\mathrm{SCP}_{\mathrm{B}}$ was found to depolarize neuron 4 and an increase in EPSP amplitude was concomitantly observed. This increase in EPSP amplitude was attributed to the spike broadening accompanying depolarization of neuron 4. Accordingly, both EPSP and spike parameters were restored to control values by repolarization of neuron 4. In contrast, the molluscan tetrapeptide FMRF-amide was found to decrease EPSP amplitude by both hyperpolarizing neuron 4 and by direct hyperpolarization and conductance increase of gland cells. In this case, repolarization of neuron 4 restored the action potential but caused only partial resto-
\end{abstract}

Received October 22, 1984; Revised March 25, 1985;

Accepted April 10, 1985

\footnotetext{
${ }^{1}$ We thank Erwin Shibata, Ken Lukowiak, and Don Murphy for comments on experimental design and on the manuscript, and Rae Barolet for manuscript preparation. This work was supported by Medical Research Council (Canada) Grants MA-7619 and MT-7619 and by grants from The Alberta Heritage Foundation for Medical Research. C.J.C. was an Alberta Heritage Foundation Medical Research summer student.

${ }^{2}$ To whom correspondence should be addressed.
}

ration of the EPSP amplitude. We conclude that the neuroglandular synapse of Helisoma shows plasticity under a variety of physiological and pharmacological conditions which involve changes of presynaptic action potential parameters.

The cellular mechanisms of synaptic plasticity have been the focus of many studies that have exploited model nervous systems varying from the hippocampus to isolated neurons in culture. In the case of molluscan ganglia, studies have ranged from investigating the onset of plasticity at identified synapses during ontogeny (Rayport and Camardo, 1984) to the cellular correlates of aging (Peretz et al., 1984). The freshwater snail Helisoma has provided a number of insights into synaptic organization including the innervation of a peripheral target organ, the salivary glands (Kater et al., 1978a). The synaptic plasticity of neuroglandular innervation in Helisoma is the subject of the current report.

Synaptic plasticity can occur in both the CNS and the PNS of molluscs, as exemplified by the ability of the gill withdrawal reflex in Aplysia to habituate with and without the presence of the CNS (Lukowiak, 1977; Carew et al., 1979). In many instances, however, the peripheral target organs and/or neurons are unsuitable for intracellular recording and, thus, analysis of mechanisms of peripheral plasticity can be confounded by technical considerations. The present study exploited the tractability of simultaneous recordings from the Helisoma salivary gland and its innervating neuron to examine plasticity at a peripheral molluscan synapse.

The somata of some neurons in molluscan ganglia have been shown to be electrically close enough to their terminal regions in the neuropil so that polarization of the soma can influence transmitter release. Specifically, hyperpolarization of the somata of interneurons in Aplysia reduces the amplitude of synaptic potentials on target neurons within the ganglion (Shirnahara and Perel, 1978; Shapiro et al., 1980; Shimahara, 1983). The present study examined the influence of soma potential on transmission at a synapse in the PNS of Helisoma under a number of physiological and pharmacological conditions

\section{Materials and Methods}

These studies employed specimens of albino ("red") Helisoma snails obtained from inbred laboratory cultures of 15 to $18 \mathrm{~mm}$ vertical shell height. The procedure for dissection and pinning out the salivary gland and attached buccal ganglia has been described previously (see Fig. 1 in Bahls et al., 1980). Unless described otherwise, recordings from the salivary gland were restricted to proximal cells (regions 1 and 2, Kater et al., 1978a).

For intracellular recording, ganglia and glands were pinned out in a 1-ml Plexiglas chamber on a thin layer of RTV 616 Silicone Rubber compound (General Electric). The chamber was continuously perfused with saline at 1 to $2 \mathrm{ml} / \mathrm{min}$ via two semicircular side arms of the chamber. These conditions were found to provide complete exchange of chamber saline within $1 \mathrm{~min}$.

Conventional electrophysiological techniques were employed, using glass fiber-filled micropipettes, the electrolyte being $0.75 \mathrm{mM} \mathrm{KCl}$. For recordings 
from salivary gland cells, the electrolyte was usually acidified with dilute $\mathrm{HCl}$ to $\mathrm{pH}$ 5.5. Acidification of the electrolyte in this manner has been found to provide longer-term recordings before electrodes attain prohibitively high resistances ("blockage"), a problem offen encountered with salivary gland recordings (A. G. M. Bulloch and C. J. Coates, unpublished observation) Currents were measured with a digital voltmeter (Datel DM-3100U1) by means of the current monitor on the electrode amplifier (Getting M5 or Dagan 8100). Electrodes were bridge-balanced with 3- to 5 -msec pulses, this being one to two orders of magnitude less than the time constant of the cells studied. For experiments involving repolarization of neuron 4 (e.g., Table II), the electrode was initially balanced for a current slightly greater than that used during the course of the experiment. Data were stored either on magnetic tape (Kaccal 4US FM instrumentation tape deck) or on a Nicolet 2090 digital oscilloscope. Action potential waveforms were analyzed on the Nicolet oscilloscope, whereas excitatory postsynaptic potentials (EPSPS) were analyzed either on the Nicolet or on a Brush-Gould $2200 S$ pen recorder. Records displayed in the text are either by the Brush-Gould recorder or by a Hewlett-Packard HP7470A plotter driven by a Nicolet 4094 oscilloscope reading from the Nicolet 2090 floppy discs.

Dissections and initial recordings were in normal Helisoma physiological saline (in millimolar concentration $\mathrm{NaCl}, 51.3 ; \mathrm{KCl}, 1.7 ; \mathrm{CaCl}_{2}, 4.1 ; \mathrm{MgCl}_{2}$ 1.5; HEPES, 5, at pH 7.3). In order to measure modulation of gland EPSPs it was necessary to reduce their size and prevent the generation of action potentials which invariably occurs in normal saline (e.g., Kater, 1974; Kater et al., 1978b). To this end, a series of salines containing a high divalent cation concentration was utilized. In these salines the total divalent cation concentration was maintained at $19 \mathrm{~mm}$, the majority of experiments utilizing saline containing 1,2 , or $3 \mathrm{~mm}$ calcium. The calcium concentration was varied in different experiments so as to produce the optimal size of EPSPs according to the experiment. The $\mathrm{Mg}^{2+}$ and $\mathrm{Ca}^{2+}$ concentrations were elevated with $\mathrm{MgSO}_{4}$ and $\mathrm{CaCl}_{2}$, respectively.

$\mathrm{SCP}_{\mathrm{B}}$ (Peninsula Laboratories) and FMRF-amide (Bachem) were made up fresh for each experiment from frozen aliquots.

Data are expressed as mean \pm standard deviation. Lines of best fit for regression analyses were calculated by the least squares method using a computer plotting routine supplied by $\mathrm{D}$. White.

\section{Results}

This study examined the plasticity of synaptic transmission between neuron 4 and the salvary gland of Helisoma under a number of experimental, physiological, and pharmacological conditions. Specifically, we attempted to relate the amplitude of gland EPSPs to the resting potential and action potential parameters of neuron 4 . This relationship was studied during imposed hyperpolarization of the neuron 4 soma, during spontaneous bursts of action potentials and imposed impulse trains, and during modification of synaptic transmission by peptides.

The structure and innervation of the salivary glands of Helisoma have been described previously (Kater et al., 1978a, b). Briefly, the salivary neuroeffector system is comprised of a pair of acinous glands that are innervated by two electrically coupled buccal ganglion neurons, L4 and R4. Action potentials in neuron 4 elicit large EPSPs which usually evoke action potentials in gland cells. The neuron 4 innervation of some secretory cells within each acinus is judged to be monosynaptic (Bahls et al., 1980), action potentials being propagated between cells by strong and extensive electrical coupling within the epithelium. In some preparations, electrical coupling between the two neurons 4 is sufficiently strong that recurrent excitation between the neurons occurs. In these cases, a characteristic deflection on the action potential downstroke ("delayed electrotonic interaction") appears and a complex gland EPSP is evoked (Bahls et al., 1980). In order to avoid the influence of a contralateral neuron 4 , having a membrane potential that could not be accurately controlled in this study, such preparations $(\sim 10 \%$ of total) were discarded.

Relationship of EPSP amplitude to soma potential of neuron 4. Synaptic transmission in the molluscan CNS can be modulated by the presynaptic membrane potential (see the introduction). We examined whether this relationship can be extended to the PNS, i.e., to a situation in which the synapse is relatively distant from the cell body.
The amplitude of gland EPSPs was found to be strongly influenced by the membrane potential of the neuron 4 soma. Specifically, stepwise increases of the resting potential of cell 4 were accompanied by a corresponding reduction in the amplitude of gland EPSPS (Fig. 1). Examination of a number of preparations revealed that the relationship of resting potential to EPSP magnitude was linear in the range from resting potential $(-47 \pm 6 \mathrm{mV}$ ) to about $-100 \mathrm{mV}$ (Fig. 2; mean correlation coefficient $=0.95 \pm 0.05, n=9$ ). This phenomenon is thought to be attributable to the reduction of action potential duration that occurs with increasing membrane potential (as described subsequently).

Relationship of EPSP amplitude to action potential of neuron 4. In an attempt to account for the modulation of the gland EPSP by the membrane potential of neuron 4 , we examined the dependence of the action potential parameters of neuron 4 on its membrane potential. Specifically, the action potential amplitude and half-maximal width were examined. The overshoot of neuron 4 impulses was found to show only marginal changes with changes of membrane potential (Fig. 3). As expected, the amplitude of action potentials, as measured from foot to peak, increased in direct proportion to the imposed hyperpolarization (Fig. 3). In contrast, the half-width of action potentials decreased substantially with increased membrane potential (Fig. 3). This decrease in width of the impulse was mainly due to changes occurring in its repolarizing phase. Furthermore, a consistent and linear relationship was observed between action potential half-width and gland EPSP amplitude (Figs. 3 and 4). This relationship was observed in all preparations examined, the mean regression correlation coefficient being $0.84 \pm 0.10(n=6)$. The narrowing of action potentials with increased membrane potential, rather than membrane potential per se, is thought to be the important variable predicting EPSP size in this system.

The correlation between soma potential and EPSP parameters is notable, but an alternative phenomenon could explain or confound our data. Specifically, soma hyperpolarization could cause spike block at distal branches of neuron 4 in the salivary gland. The EPSP recorded in a proximal acinus might then be reduced since the influence of EPSPs in electrically coupled distal cells will be reduced. This explanation was ruled out, however, by recordings from the most distal acini. No EPSP failures were observed, even upon marked hyperpolarization of neuron 4 . On the contrary, the EPSP amplitude was reduced by a smaller degree than that observed in more proximal acini in the same preparation, presumably because of the increased electrotonic length of distal cells from the neuron 4 soma.

Physiological role for action potential half-width/EPSP relationship. Although the modulation of EPSP size by presynaptic membrane potential is striking, during normal physiological activity the variation in presynaptic membrane potential is more restricted than under our experimental conditions. It was of interest to determine whether changes of action potential duration occur during normal physiological activity and are accompanied by changes of EPSP magnitude. We therefore examined whether spontaneous activity of neuron 4 is accompanied by variation of action potential and EPSP parameters.

Activity was recorded for variable lengths of time during which both the neuron 4 action potential half-width and the gland EPSP amplitude were monitored. Spontaneous activity of neuron 4 in the isolated buccal ganglia typically consists of bursts of impulses of variable length occurring at 2 to 3 bursts/min. Examination of individual action potential bursts revealed that modulation of EPSPS does indeed occur during normal activity (Fig. 5). In particular, when the interval between successive action potentials was $<2 \mathrm{sec}$, facilitation of the second EPSP was consistently observed (Fig. 5). Examination of the action potential in such cases revealed a lengthened half-width of the second impulse. In monitoring trains of successive bursts, a strong correlation was observed between the EPSP amplitude and action potential half-width (Fig. 5). Thus, the action potential/EPSP relationship described above can be extended 

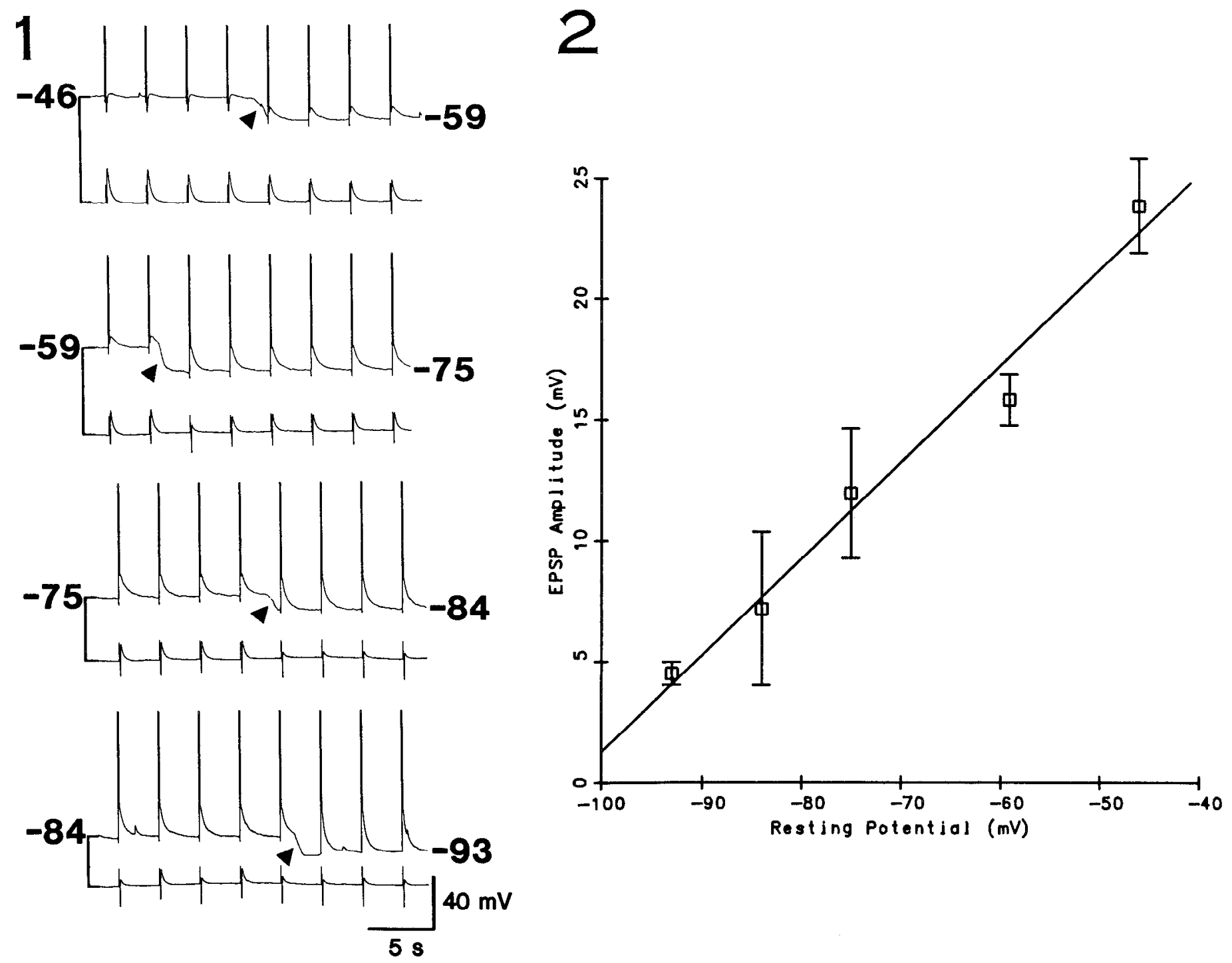

Figure 1. Modulation of gland EPSP amplitude by the membrane potential of neuron 4 . Segments of a continuous recording from neuron L4 and an ipsilateral gland cell are shown, action potentials being elicited with 10-msec pulses at 2.5-sec intervals (no synaptic facilitation occurs at this frequency; see the text and Figs. 5 and 6$)$. The neuron was initially at resting potential $(-46 \mathrm{mV}$ ) and was hyperpolarized at the arrowheads by $0.1-\mathrm{nA}$ increments to the indicated holding potentials. The electrode was initially bridge-balanced for the maximum holding current, $0.4 \mathrm{nA}$, and its balance did not differ for the smaller currents. The records are continuous except in the case of the lower pair, between which four action potentials have been deleted. The preparation was bathed in $3 \mathrm{~mm} \mathrm{Ca}^{2+}, 16 \mathrm{~mm} \mathrm{Mg}^{2+}$ saline.

Figure 2. Relationship of neuron 4 membrane potential to amplitude of the gland EPSP. The experimental paradigm is described in Figure 1, which shows examples of records. Correlation coefficient $=0.98$, slope $=0.40$. Each point is the mean \pm standard deviation $(n=4)$.

to spontaneous activity occurring at a more or less constant neuron 4 membrane potential.

In order to examine the action potential/EPSP relationship at constant resting potential more rigorously, the activity of neuron 4 was suppressed by prolonged hyperpolarization, and spike trains were evoked either by release of the hyperpolarization or by a depolarizing pulse upon release of the hyperpolarization. This treatment produced a steady train of action potentials which showed gradıal broadening (Fig. 6). Successively broader action potentials were accompanied by a sequence of correspondingly increased EPSPS. As before, the EPSP amplitude was strongly correlated with action potential half-width (mean correlation coefficient $=0.97 \pm$ $0.02, n=6)$.

Although the linear correlation between soma action potential and gland EPSP size was consistent from preparation to preparation in all of the above studies, the numerical relationship of the two pararneters was somewhat variable. Specifically, the slope of the EPSP amplitude/half-width relationship varied from 0.08 to 3.02 in different preparations (mean slope $=1.51 \pm 0.98, n=13$ ). The slope value is an important parameter to be considered when evaluating the dependency of EPSP size upon action potential halfwidth in any given preparation because it provides a measure of the sensitivity of the synapse with respect to changes in the neuron 4 action potential. The basis of this differential sensitivity between preparations is unknown.

Peptidergic modulation of neuroglandular transmission. A number of roles have been postulated for both classical transmitters and peptides in the modulation of synaptic efficacy. We further examined the significance of the relationship between neuron 4 action potential half-width and gland EPSP magnitude by testing its possible utilization by two peptides known to modulate synapses in molluscs, i.e. $\mathrm{SCP}_{\mathrm{B}}$ and FRMF-amide.

Bath applications of $\mathrm{SCP}_{\mathrm{B}}$ to the buccal ganglia/salivary gland preparation reliably caused an increased amplitude of gland EPSPS. In all preparations, $\mathrm{SCP}_{\mathrm{B}}$ caused marked effects at $10^{-6}$ to $10^{-7} \mathrm{M}$, whereas the lowest concentration at which synapse modulation 

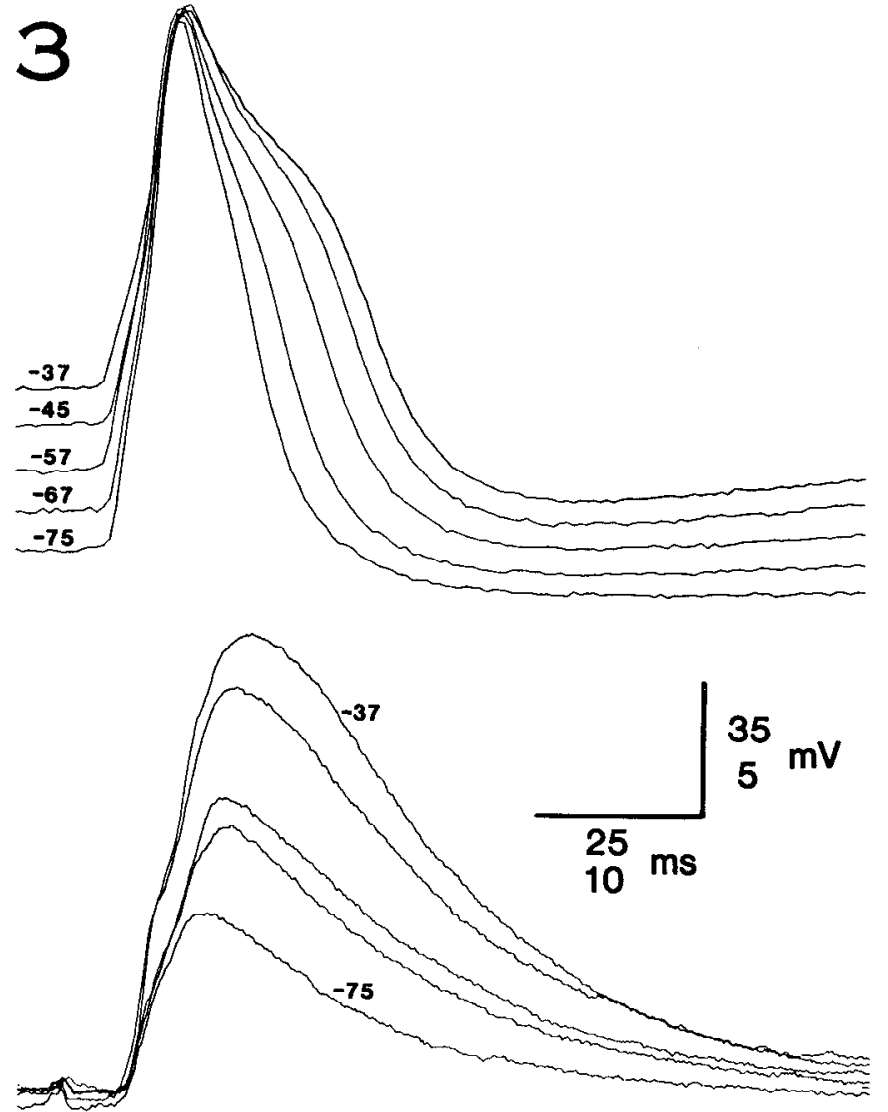

Figure 3. Reduction of action potential half-width of neuron 4 and of the amplitude of gland EPSPs by hyperpolarization of the neuron 4 soma. Neuron 4 was hyperpolarized from its initial resting potential $(-37 \mathrm{mV})$ by $0.1-\mathrm{nA}$ current increments to the holding potentials indicated. As a result of the step hyperpolarizations, the action potential in neuron 4 becomes progressively narrower and the corresponding EPSP in the gland cell becomes smaller. These action potentials occurred spontaneously. In the event of a burst of impulses, the first was chosen for measurement so as not to be influenced by spike broadening that occurs during bursts or trains (Figs. 5 and 6). The waveforms depicted here are single examples at each holding potential, and all of the data points from this preparation are depicted graphically in Figure 4. The preparation was bathed in $2 \mathrm{~mm} \mathrm{Ca}^{2+}, 17 \mathrm{mM} \mathrm{Mg}^{2+}$ saline. Note the different time scales (also in Figs. 6 to 8 ) which were chosen for maximum clarity, each pair of traces beginning together.

Figure 4. Relationship of neuron 4 action potential half-width to gland $\mathrm{EPSP}$ amplitude. This relationship is linear with correlation coefficient $=0.94$, slope $=0.75$. Same preparation as depicted in Figure 3 .

could be observed was $10^{-9} \mathrm{M}$. This modulation of neurotransmission was accompanied by increased activity of neuron 4 . The occurrence of such activity confounded measurements of action potential and EPSP parameters since closely occurring impulses produce spike broadening and EPSP facilitation (Figs. 5 and 6). For this reason, neuron 4 was hyperpolarized sufficiently to suppress spontaneous activity during $\mathrm{SCP}_{\mathrm{B}}$ application. Under these conditions $\mathrm{SCP}_{\mathrm{B}}$ caused a depolarization of neuron 4 and action potentials broadened in parallel with the increased EPSP amplitude (Table I, Fig. 7). I hat this modulation of the gland EPSP is solely attributable to depolarization of neuron 4 was directly demonstrated by repolarization of neuron 4 during $\mathrm{SCP}_{\mathrm{B}}$ application. Both the spike width and gland EPSP were consistently restored to within $\pm 10 \%$ of control values by neuron 4 repolarization (Table II) in all preparations tested $(n=6)$. Thus, $\mathrm{SCP}_{\mathrm{B}}$ modulates neuroglandular transmission by utilizing the action potential duration/EPSP amplitude relationship. In contrast to FMRF-amide (below), no direct postsynaptic changes (e.g., input impedance and resting potential of gland cells) due to $\mathrm{SCP}_{\mathrm{B}}$ application (Table I) were observed.

The molluscan tetrapeptide FRMF-amide was also found to modulate the efficacy of neurotransmission on the gland. In common with $\mathrm{SCP}_{\mathrm{B}}$, FMRF-amide consistently altered neuroglandular efficacy at concentrations of $10^{-6}$ to $10^{-7} \mathrm{M}$ threshold effects being observed at $10^{-9} \mathrm{M}$. The effects of FMRF-amide, however, were more complex than that of $\mathrm{SCP}_{\mathrm{B}}$, since it appears to act directly upon both neuron 4 and the gland cells. Specifically, FMRF-amide application to the bath caused a hyperpolarization of both neuron 4 and the gland colls (Fig. 8). Concurrently, the gland EPSP showed a drastic reduction in amplitude which was associated with a moderate decrease of neuron 4 action potential half-width (Table I). The increase of gland cell membrane potential implied a conductance change and, consequently, we examined the input impedance of gland cells during FMRF amidc application. Thus, FMRF-amide was found to decrease the input impedance of gland cells (Table 1). Since this loss of input impedance will itself reduce EPSP size, this augments the reduction of EPSP amplitude attributable to hyperpolarization of neuron 4. Accordingly, repolarization of neuron 4 caused only partial restoration of the gland EPSP amplitude despitc recovery of spike half-width to within $\pm 10 \%$ of its control value in all preparations tested $(n=5)$. Thus, FMRF-amide exhibits actions both preand post-synaptically in this system.

\section{Discussion}

The mechanisms underlying physiological and behavioral plasticity are a major current focus of neurobiology. The identified neurons of the freshwater snail Helisoma have been utilized for studies of synaptic connectivity and motor pattern generation (e.g., Kater, 1974), and neuronal regeneration (e.g., Bulloch, 1985). The current report extends this range of studies by examining synaptic plasticity in the PNS. In particular, we examined modulation of synaptic efficacy in the PNS by physiological manipulations of the central cell body. 


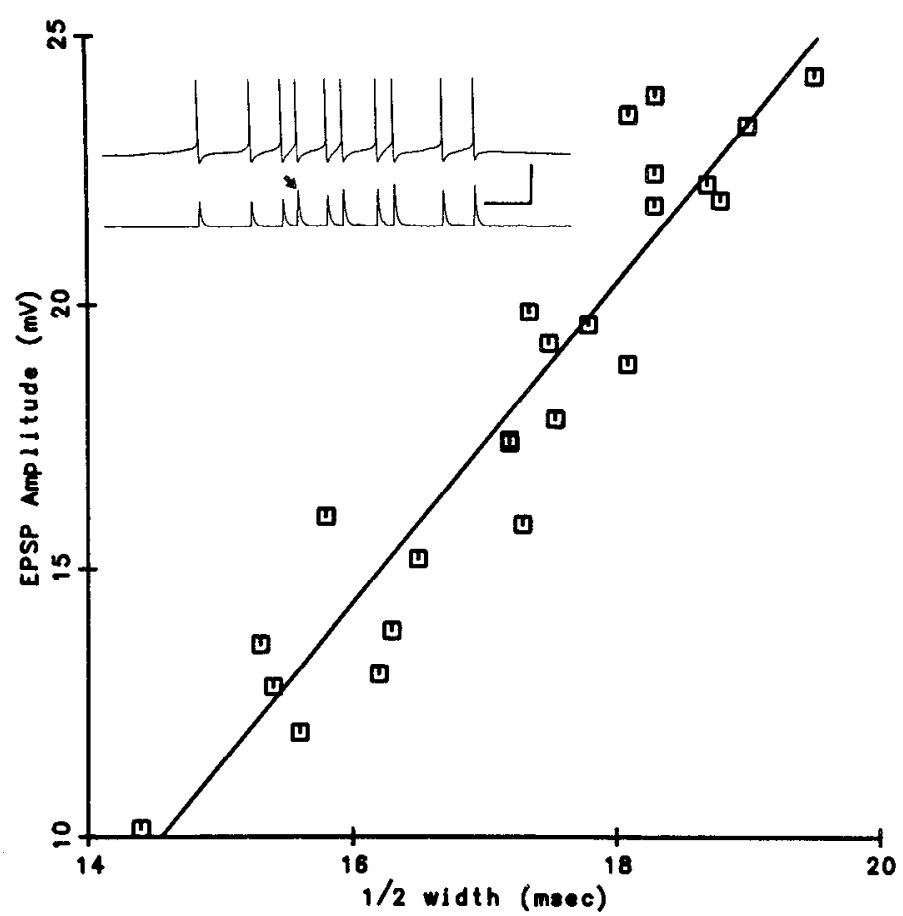

Figure 5. Modulation of gland EPSP amplitude during spontaneous activity in neuron 4. A spontaneous burst of action potentials in neuron R4 and the corresponding EPSPs in an ipsilateral gland cell are shown as the inset (upper left). Note the EPSP facilitation when spikes occur at <2-sec intervals (e.g., at arrow). The graph depicts EPSP amplitude versus neuron 4 action potential half-width during a series of three such bursts of action potentials containing a total of 24 impulses. Correlation coefficient $=0.94$, slope $=$ 2.20. The preparation was bathed in $1 \mathrm{~mm} \mathrm{Ca}{ }^{2+}, 18 \mathrm{mM} \mathrm{Mg}^{2+}$ saline. Calibration bars for insct: $40 \mathrm{mV}$ (upper), $20 \mathrm{mV}$ (lower), and $2.5 \mathrm{scc}$.
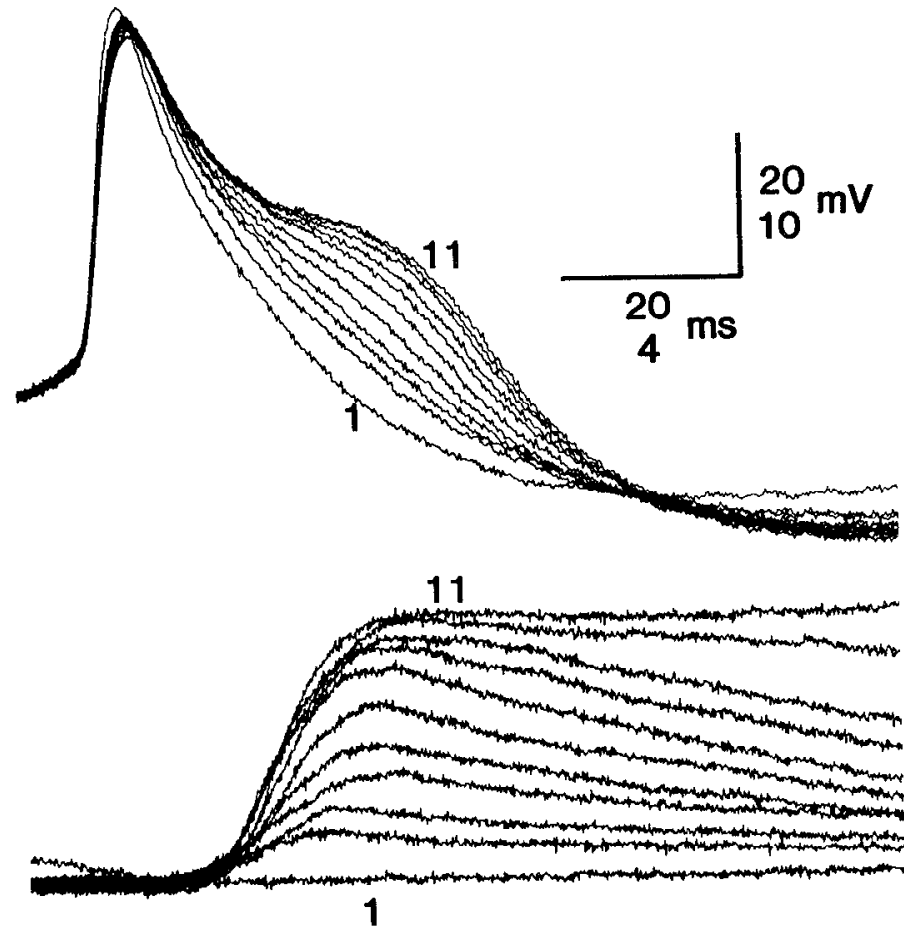

Figure 6. Action potential broadening and facilitation of gland EPSPS during an impulse train. This train of 11 action potentials in a neuron 4 was driven by a depolarizing $0.1 \mathrm{nA}$ current pulsc. Preceding the depolarization, spontaneous action potential activity was suppressed by a 20-sec, 0.1-nA hyperpolarization. The EPSP amplitude is linearly related to action potential half-width, correlation coefficient $=0.98$, slope $=0.93$. The preparation was bathed in $1 \mathrm{mM} \mathrm{Ca}^{2+}, 18 \mathrm{mM} \mathrm{Mg}^{2+}$ saline.
Our results demonstrate that synapses in the molluscan PNS can be controlled by the soma potential of an effector neuron. The ability of presynaptic membrane potential to influence transmitter release has been demonstrated in a number of preparations, but with differing results which are compared in Table III. The situation in the Helisoma neuroglandular preparation resembles that of interneuroneffector neuron synapses within the Aplysia abdominal ganglion. In the case of the Aplysia abdominal interneuron L10, hyperpolarization reduces both the duration and the amplitude of action potentials (Shapiro et al., 1980), whereas in Helisoma buccal neuron 4, hyperpolarization reduces only the duration of impulses (which increase in amplitude as measured from holding potential, Fig. 4). In contrast, pleural ganglion interneurons in Aplysia exhibit reduction of spike amplitude without reduced half-width upon hyperpolarization, this being attributable to decreased inactivation of early outward current (Shimahara, 1983). The differences between molluscan systems are further exemplified by the linear relationships observed in the current report between soma membrane potential and EPSP amplitude as opposed to the S-shaped relationship observed in the case of CNS synapses in other molluscs over comparable voltage ranges (Shimahara and Peretz, 1978; Shapiro et al., 1980). The situation is lurther diversified by consideration of the squid giant synapse and the crayfish neuromuscular junction. In the former case, presynaptic depolarization decreases transmitter output, apparently due to decreased amplitude of action potentials for which the half-width is unchanged (Miledi and Slater, 1966; Charlton and Bittner, 1978). Recordings from motoneuron branches close to crustacean muscles have shown that prolonged depolarization can enhance transmitter output, despite reduced spike amplitude, apparently because of enhanced steady-state $\mathrm{Ca}^{2+}$ current (Wojtowicz and Atwood, 1984). Thus, the mechanisms of synaptic plasticity are diverse, even within a phylum, and require individual characterization.

The strong linear correlation between the neuron 4 half-width and gland EPSP amplitude in Helisoma indicates that action potential duration is the predominant factor determining transmitter release at this peripheral synapse. The soma action potential half-width therefore appears to provide a reasonable approximation of the duration of excitation at the terminals. The form of terminal excitation, i.e., action potential invasion or passive spread of current from axonal action potentials, is unknown in gastropod molluscs but could resemble the active invasion of terminals observed at the squid giant synapse (Bullock and Hagiwara, 1957).

The ability to control transmitter release in the PNS by the soma potential is unexpected since, unlike the situation for an interneuron in the CNS, the salivary glands might be expected to be electrically distant from the neuron 4 soma. The length constants of molluscan neurons, however, are often cxtraordinarily long (Horn, 1978). The length constant for Helisoma neuron 4 is unknown but may be

TABLE 1

Modulation of neuroglandular transmission by $\mathrm{SCP}_{B}$ and FMRF-amide Measurements were made at maximal effect of peptides ( 1 to 3 min tor $\mathrm{SCP}_{\mathrm{B}}, 2$ to $4 \mathrm{~min}$ for FMRF-amide). During $\mathrm{SCP}_{\mathrm{B}}$ application neuron 4 was hyperpolarized by a current sufficient to block spontaneous action potentials (0.1 to $0.2 \mathrm{nA}$ ).

\begin{tabular}{lcc}
\hline Parameter & $\mathrm{SCP}_{\mathrm{B}}\left(10^{-7} \mathrm{M}\right)$ & FMRF-amide $\left(10^{-7} \mathrm{M}\right)$ \\
Gland EPSP magnitude & $+45 \pm 12 \%$ & $-44 \pm 13 \%$ \\
& $(n=6)^{\mathrm{a}}$ & $(n=5)$ \\
Neuron 4 spike half-width & $+27 \pm 12 \%$ & $-17 \pm 5 \%$ \\
& $(n=6)$ & $(n=5)$ \\
Neuron 4 membrane po- & $+5.8+2.0 \mathrm{mV}$ & $-7.8 \pm 5.1 \mathrm{mV}$ \\
tential & $(n=6)$ & $(n=5)$ \\
Gland cell membrane po- & No change & $-7.0 \pm 2.8 \mathrm{mV}$ \\
tential & $(n=6)$ & $(n=19)$ \\
Gland cell input imped- & No change & $-29 \pm 20 \%$ \\
ance & $(n=5)$ & $(n=24)$ \\
\hline
\end{tabular}

${ }^{a} n=$ number of preparations tested. 


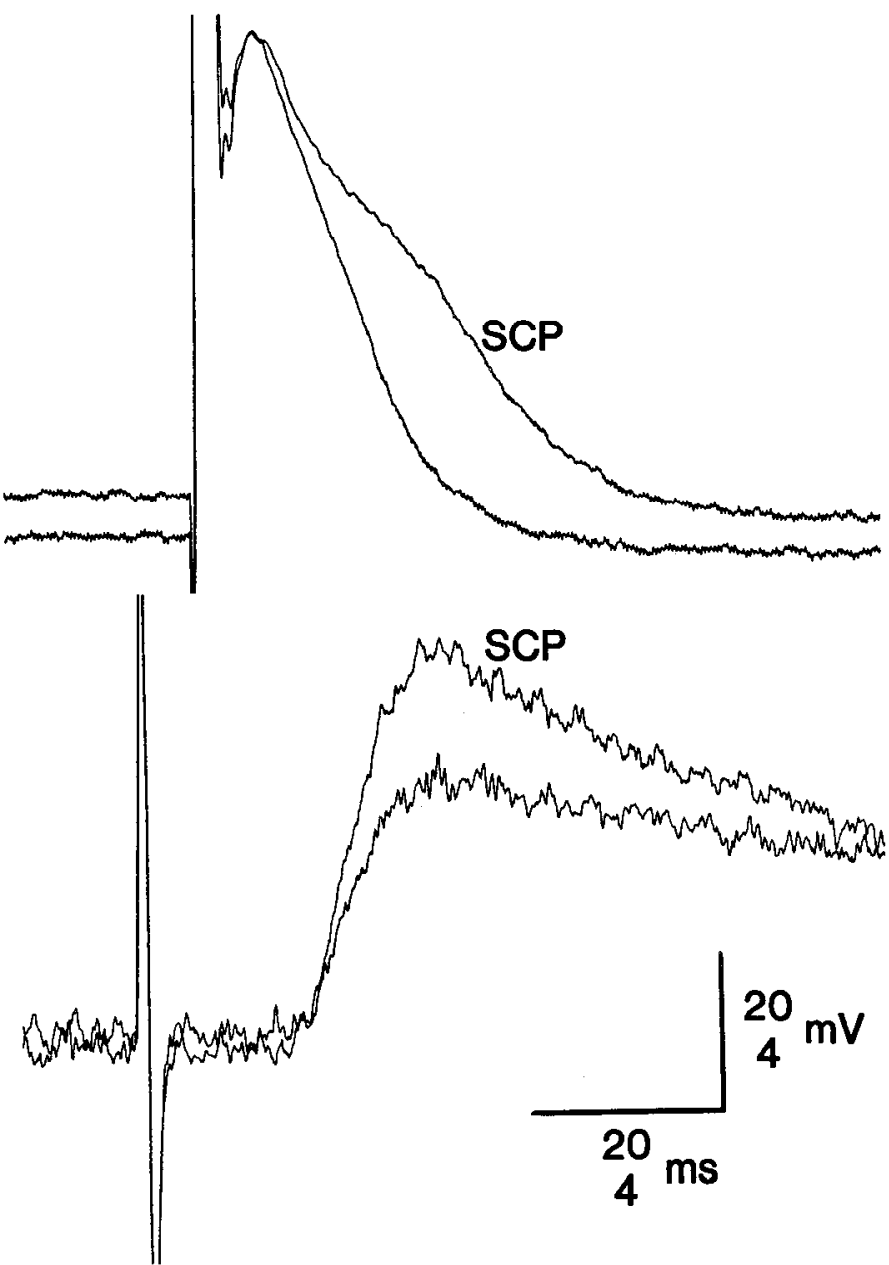

Figure 7. Modulation of neuroglandular Iransmission by $\mathrm{SCP}_{\mathrm{B}}$. Examples of action potentials (stimulated by 3 -msec pulses every $3 \mathrm{sec}$ ) and EPSPS immediately before and $1 \mathrm{~min}$ after bath application of $10^{-7} \mathrm{MSCP}$. In this preparation neuron 4 was hyperpolarized by a steady $0.25-n A$ current to suppress spontaneous impulse activity. $\mathrm{SCP}_{\mathrm{B}}$ application caused a 5- to 6$\mathrm{mV}$ depolarization and subsequent spike broadening. Seven control action potentials (taken at 30-sec intervals) and nine during the first 3 min of SCP depolarization showed a spike half-width/EPSP linear correlation of coefficient 0.86 , slope $=0.28$. The preparation was bathed in $2 \mathrm{mM} \mathrm{Ca}^{2+}, 17 \mathrm{mM} \mathrm{Mg}^{2+}$ saline.

approximated given the average coupling coefficient of the two neurons 4 (0.54, measured soma-to-soma, Murphy et al., 1983), the soma-to-soma distance $(1 \mathrm{~mm})$, and assurning exponential decay between the somata without decrement at the electrical synapse. This calculation, which must provide a minimum indication of the length constant (given that some decay probably occurs across the electrical junction), provides a value of $1.5 \mathrm{~mm}$. The distance from neuron 4 soma to the proximal gland acini is about $3 \mathrm{~mm}$. These calculations give credibility to the notion that soma potential can influence the terminal potential in this system. Furthermore, soma potential was found to influence the EPSP in the most distal acini of the gland, which are $6 \mathrm{~mm}$ from the gland 4 soma. In the larger opistobranch molluscs like Aplysia, length constants are greater still ( $5 \mathrm{~mm}$, Horn, 1978); thus, soma potentials in these animals can probably control terminals at least up to $1 \mathrm{~cm}$.

The ionic mechanism underlying the dependency of spike duration upon membrane potential and frequency in neuron 4 is as yet unknown but presumably involves increased $\mathrm{Ca}^{2+}$ flux due to decreased outward currents and/or increased inward currents. In the case of the Aplysia neuron L10, spike broadening and enhanced release have been shown to involve both a decreased spike $\mathrm{K}^{+}$ conductance and an increased steady-state $\mathrm{Ca}^{2+}$ current (Shapiro et al., 1980).

Neuromodulatory effects of peptides have been implicated in a variety of systems (Hokfelt et al., 1980; Strumwasser, 1982; Iversen et al., 1983). A number of neuronal properties can be influenced by peptides, including membrane potential and action potential properties (e.g., Pittman, 1980). With the exception of studics in tissue culture (e.g., MacDonald and Nelson, 1978), the mechanisms by which peptides alter synaptic efficacy in the vertebrates are often unclear. Certain invertebrates offer preparations which enable study of peptidergic modulation of synapses at the level of single cells (e.g., Hooper and Marder, 1984; Scheller et al., 1984). In the mollusc, for example, cardioactive peptides are known to modulate neuromuscular synapses. For example, $\mathrm{SCP}_{\mathrm{B}}$ increases the rate of relaxation of nerve-stimulated twitches in Helix (Lloyd, 1980a, b) and enhances muscle contractions in Aplysia (Lloyd et al., 1984). Additionally, FMRF-amide enhances the amplitude of muscle contractions and excitatory junction potentials in Aplysia (Richmond et al., 1984).

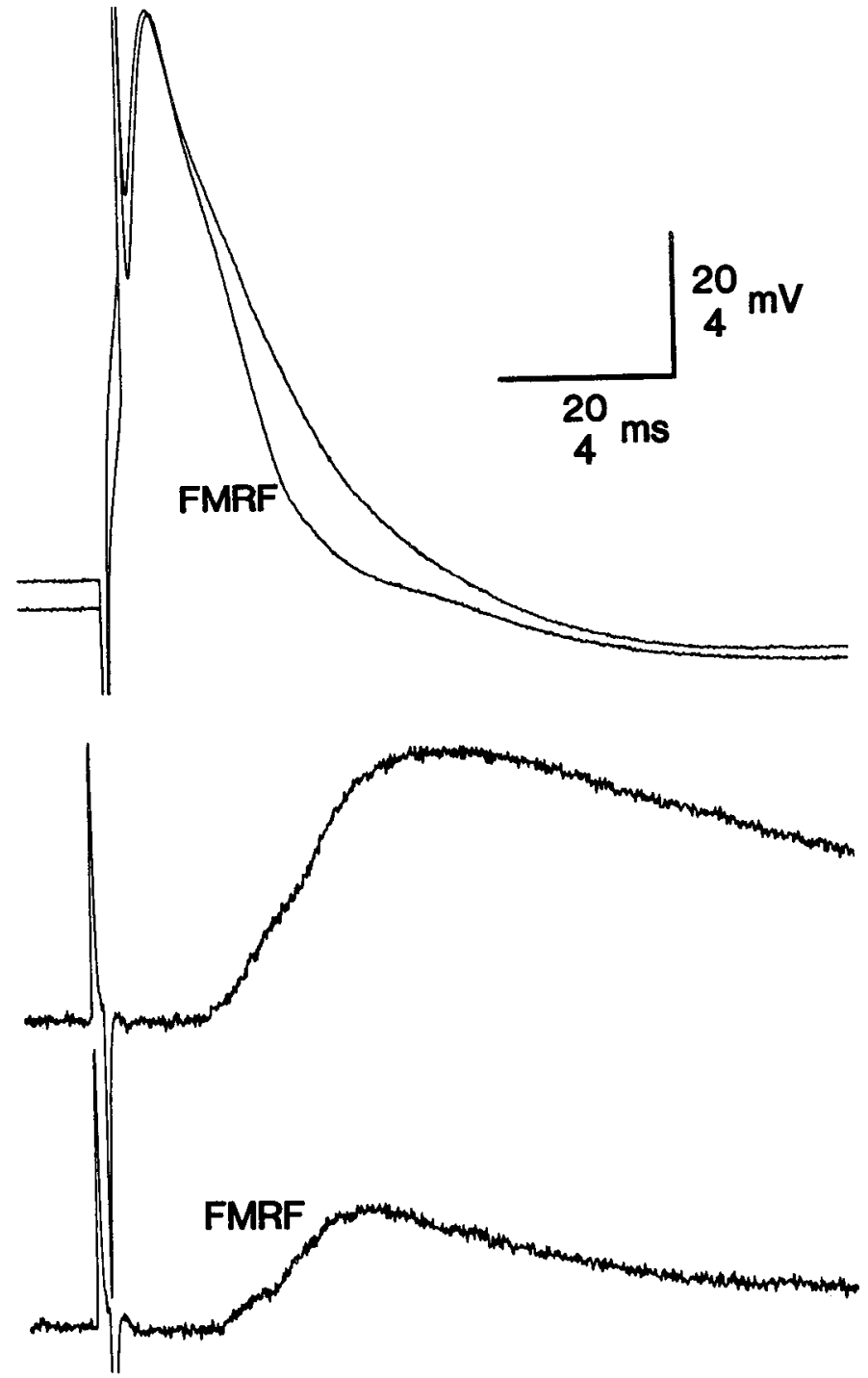

Figure 8. Modulation of neuroglandular transmission by FMRF-amide. Examples of action potentials (stimulated by $3-\mathrm{msec}$ pulses every $5 \mathrm{sec}$ ) and EPSPS immediately before and $2 \mathrm{~min}$ after an application of $10^{-7} \mathrm{M}$ FRMFamide. Neuron 4 was initially at resting potential, FRMF-amide causing a 5$\mathrm{mV}$ and $9-\mathrm{mV}$ hyperpolarization of neuron 4 and the gland cell, respectively. The decreased amplitude of the gland EPSP is due to both narrowing of the neuron 4 action potential and decreased input impedance of the gland cell (See Table II). The preparation was bathed in $3 \mathrm{mM} \mathrm{Ca}^{2+}, 16 \mathrm{mM} \mathrm{Mg}^{2+}$ saline. 
TABLE ॥

Reversal of $S C P_{B}$ and partial reversal of FMRF-amide neuroglandular modulation by repolarization of neuron 4

In part $A$, neuron 4 was initially hyperpolarized with a $0.2-n A$ current (column 2 data). $\mathrm{SCP}_{\mathrm{B}}$ caused a 7 -mV depolarization, measurements being made after 1 to $3 \mathrm{~min}$ of peptide application (column 3). Subsequently, neuron 4 was repolarized to its former holding potential (total electrode current, $0.34 \mathrm{nA}$ ) and new measurements were made (column 4). Finally, recovery measurements were made at the initial holding potential after 5 min of washout (column 5). In part B, neuron 4 was initially at resting potential (column 2). FMRF-amide caused a 4-mV hyperpolarization of neuron 4 , data being taken after 2 to 4 min of peptide application (column 3). Neuron 4 was subsequently repolarized to its former membrane potential with a 0.06-nA current (column 4). Finally, recovery measurements were made at resting potential after 5 min of washout.

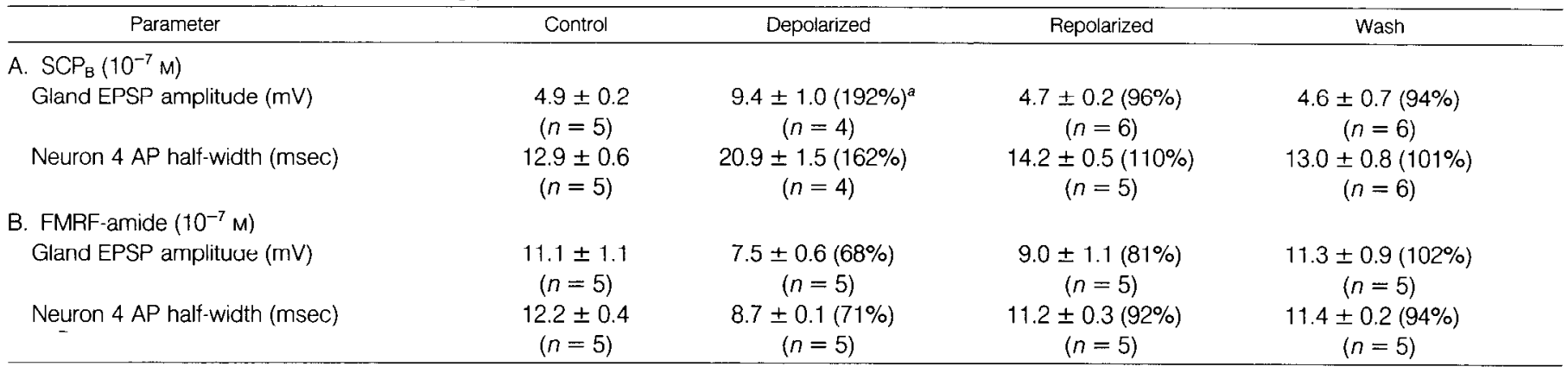

${ }^{a}$ Percentage values are with respect to controls.

TABLE III

Regulation of synaptic efficacy by presynaptic membrane potential

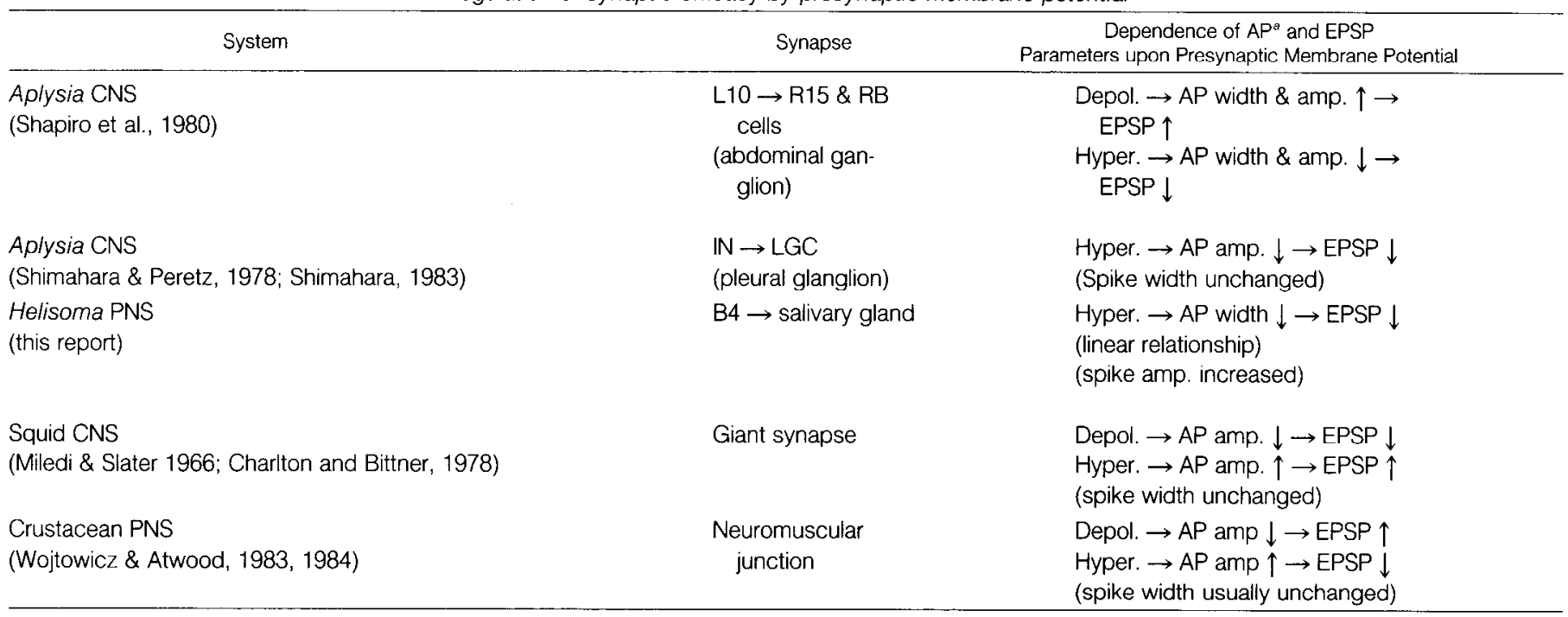

${ }^{a}$ AP, action potential; Depol., depolarization; amp., amplitude; Hyper., hyperpolarization.

The evidence favors a postsynaptic locus for action of both $\mathrm{SCP}_{\mathrm{B}}$ and FMRF-amide on molluscan muscle, although presynaptic actions have not been ruled out.

The present observations of a peptidergic involvement in synaptic plasticity have revealed two mechanisms for peptide modification of synaptic efficacy in molluscs. We have shown that $\mathrm{SCP}_{\mathrm{B}}$ facilitates synaptic transmission by virtue of its ability to depolarize neuron 4 , i.e., by utilizing the action potential duration/EPSP amplitude dependency we demonstrated physiologically. In contrast, FMRFamide has both pre- and postsynaptic actions. The presynaptic hyperpolarization of neuron 4 utilizes the same mechanism, but EPSP size is further reduced by a postsynaptic decrease of input impedance. Thus, peptides such as FMRF-amide and $\mathrm{SCP}_{\mathrm{B}}$ can express part or all of their action by utilizing a simple presynaptic membrane potential/postsynaptic EPSP amplitude relationship. This is in contrast to the membrane potential-independent modulation of action potentials in cultured chick sensory neurons by enkephalin (Mudge et al., 1979). It will be of interest to determine whether the alternative means by which presynaptic membrane potential can modulate synaptic efficacy (Table III) are exploited by neuromodulators in other preparations.
It will be important to determine whether a physiological role exists for the pharmacological responses to peptides that we have demonstrated in this study. To this end, studies have been initiated regarding the localization of FMRF-amide and $\mathrm{SCP}_{\mathrm{B}}$ neurons and fituers in Helisoma. Preliminary immunofluorescence studies have indicated the presence of both FMRF-amide-like and $\mathrm{SCP}_{\mathrm{B}}$-like fibers on the Helisoma salivary gland (A. D. Murphy and K. Lukowiak. unpublished observation). This system may therefore provide the opportunity to further evaluate the peptidergic modulation of synapses in terms of all the participating neurons.

\section{References}

Bahls, F., S. B. Kater, and R. W. Joyner (1980) Neuronal mechanisms for bilateral coordination of salivary gland activity in Helisoma. J. Neurobiol. 11: 365-739

Bulloch, A. G. M. (1985) Development and plasticity of the molluscan nervous system. In The Mollusca Neurobiology and Behavior, Part I, A. O. D. Willows, ed., Vol 8, pp. 335 410, Academic Press, Inc., Now York.

Bullock, T. H., and S. Hagiwara (1957) Intracellular recording from the giant synapse of the squid. J. Gen. Physiol. 40: 565-577.

Carew, T. J., V. F. Castellucci, J. H. Byrne, and E. R. Kandel (1979) Quantitative analysis of relative contribution of central and peripheral 
neurons to gill withdrawal reflex in Aplysia californica. J. Neurophysiol. 42 : 497-509.

Charlton, M. P., and G. D. Bittner (1978) Presynaptic potentials and facilitation of transmitter release in the squid giant synapse. J. Gen. Physiol. 72: 487512 .

Hokfelt, T., O. Johansson, A. Ljungdahl, J. M. Lundberg, and M. Schultzberg (1980) Peptidergic neurones. Nature 284: 515-521.

Hooper, S. L., and E. Marder (1984) Modulation of a central pattern generator by two neuropeptides, proctolin and FMRFamide. Brain Res. 305: 186191.

Horn, R. (1978) Propagating calcium spikes in an axon of Aplysia. J. Physiol. (Lond.) 281: 513-534.

Iversen, L. L., S. D. Iversen, and S. H. Snyder (1983) Handbook of Psychopharmacology, Vol. 16: Neuropeptides, Plenum Press, New York.

Kater, S. B. (1974) Feeding in Helisoma trivolvis: The morphological and physiological bases of a fixed action pattern. Am. Zool. 14: 1017-1036.

Kater, S. B., A. D. Murphy, and J. R. Rued (1978a) Control of the salivary glands of Helisoma by identified neurones. J. Exp. Biol. 72: 91-106.

Kater, S. B., J. R. Rued, and A. D. Murphy (1978b) Propagation of action potentials through electrotonic junctions in the salivary glands of the pulmonate mollusc, Helisoma trivolvis. J. Exp. Biol., 72: 77-90.

Lloyd, P. E. (1980a) Modulation of neuromuscular activity by 5-hydroxytryptamine and endogenous peptides in the snail Helix aspersa. J. Comp. Physiol. A 139:333-339

Lloyd, P. E. (1980b) Mechanisms of action of 5-hydroxytryptamine and endogenous peptides in the snail, Helix aspersa. J. Comp. Physiol. A 139: $341 \cdot 347$.

Lloyd, P. E., I. Kupferman, and K. R. Weiss (1984) Evidence for parallel actions of a molluscan neuropeptide and serotonin mediating arousal in Aplysia. Proc. Nall. Acad. Sci. U. S. A. 81: 2934-2937.

Lukowiak, K. (1977) CNS control of the PNS-mediated gill withdrawal reflex and its habituation. Can. J. Physiol. Pharmacol. 55: 1252-1262

MacDonald, R. L., and P. G. Nelson (1978) Specitic opiate-induced depression of transmitter release from dorsal root ganglion cells in culture. Science 199: $1449-1450$.

Miledi, R., and C. R. Slater (1966) The action of calcium on neuronal synapses in the squid. J. Physiol. (Lond.) 184: 473-498.
Mudge, A. W. S. E. Leeman, and G. D. Fischbach (1979) Enkephalin inhibits release of substance $P$ from sensory neurons in culture and decreases action potential duration. Proc. Natl. Acad. Sci. U. S. A. 76: 526-530.

Murphy, A. D. R. D. Hadley, and S. B. Kater (1983) Axotomy-induced parallel increases in electrical and dye coupling between identified neurons of Helisoma. J. Neurosci. 3: 1422-1429.

Peretz, B., A. Romanenko, and W. Markesbery (1984) Functional history of two motor neurons and the morphometry of their neuromuscular junctions in the gill of Aplysia: Evidence for differential ageing. Proc. Natl. Acad. Sci. U. S. A. 81: 4232-4236.

Pittman, Q. J. (1980) Peptide modulation of neuronal electrical responses. In Advances in Physiological Sciences. Vol. 14: Endocrinology, Neuroendocrinology, Neuropeptides-II, E. Stark, G. B. Makara, B. Halasz, and G. Rappay, eds., pp. 231-241, Pergamon Press, Budapest.

Rayport, S. G., and J. S. Camardo (1984) Differential emergence of cellular mechanisms mediating habituation and sensitization in the developing Aplysia nervous system. J. Neurosci. 4: 2528-2532.

Richmond, J. E., A. G. M. Bulloch, and K. L. Lukowiak (1984) Peptidergic modulation of a neuromuscular junction in Aplysia. Soc. Neurosci. Abstr. 10: 690 .

Scheller, R. H., R. -R. Kaldany, T. Kreiner, A. C. Mahon, J. R. Nambu, M Schaefer, and R. Taussig (1984) Neuropeptides: Mediators of behavior in Aplysia. Science 225: 1300-1308.

Shapiro, E., V. F. Castellucci, and E. R. Kandel (1980) Presynaptic membrane potential affects release in an identified neuron of Aplysia by modulating $\mathrm{Ca}^{2+}$ and $\mathrm{K}^{+}$currents. Proc. Natl. Acad. Sci. U. S. A. 77: 629-633.

Shimahara, T. (1983) Presynaptic modulation of transmitter release by the early outward potassium current in Aplysia. Brain Res. 263: 51-56.

Shimahara, T., and B. Peretz (1978) Soma potential of an interneurone controls transmitter release in a monosynaptic pathway in Aplysia. Nature 273: $158-160$

Strumwasser, F. (1982) Introduction: Comparative neurobiology of peptidergic systems. Fed. Proc. 41: 2919-2922.

Wojtowicz, J. M., and H. L. Atwood (1984) Presynaptic membrane potential and transmitter release at the crayfish neuromuscular junction. J. Neurophysiol. 52: 99-113. 